\title{
Comparing the effect of different spine and leg designs for a small bounding quadruped robot
}

\author{
Peter Eckert ${ }^{1,2}$, Alexander Spröwitz ${ }^{1,3}$, Hartmut Witte ${ }^{4}$ and Auke Jan Ijspeert ${ }^{1}$
}

\begin{abstract}
We present Lynx-robot, a quadruped, modular, compliant machine. It alternately features a directly actuated, single-joint spine design, or an actively supported, passive compliant, multi-joint spine configuration. Both spine configurations bend in the sagittal plane. This study aims at characterizing these two, largely different spine concepts, for a bounding gait of a robot with a three segmented, pantograph leg design. An earlier, similar-sized, bounding, quadruped robot named Bobcat with a two-segment leg design and a directly actuated, single-joint spine design serves as a comparison robot, to study and compare the effect of the leg design on speed, while keeping the spine design fixed. Both proposed spine designs (single rotatory and active and multi-joint compliant) reach moderate, self-stable speeds.
\end{abstract}

\section{INTRODUCTION}

The animal world can pose as the role model for locomotion of quadruped-robots and provide valuable insight into construction guidelines for mechanics as well as the corresponding control structure (see Section II). Research on quadruped robots towards high dynamic locomotion has led to many different robotic platforms (Table I). Robots like BigDog [1] show rough terrain locomotion with a strong focus on balance and locomotion control, and a high energy consumption. On the other hand, passive and active compliant elements potentially allow for dynamic gaits with less need for high-bandwidth control, but equally stable locomotion. Tsujita and Miki [2] presented a quadruped robot with a hydraulically actuated spine. It was able to perform stable locomotion in different motion patterns. With focus on leg design, robot platforms such as Scout II [3], Cheetah-cub robot [4], and Bobcat-robot[5] perform in different classes of speed and cost of transport (Table I). Videos published by the development teams show the MIT Cheetah $(v=6.1 \mathrm{~m} / \mathrm{s}$ [6]) and the Boston Dynamics Cheetah $(v=12.6 \mathrm{~m} / \mathrm{s} /$ no other scientific data available) or the Boston Dynamics Wildcat $(7.15 \mathrm{~m} / \mathrm{s} /$ no other scientific data available) give new outlines of maximum speed reachable in legged-robotics. On the other hand, few of these machines feature the design rules derived from small mammals [7] extensively. Small mammals are in size comparable to the investigated robots and the findings analyzing this animal group are thus used in the current development process. Most often applied gaits are not the highly dynamical ones like gallop, bound, or half-bound but trot or walk, even though bounding gaits

\footnotetext{
1 Biorobotics Laboratory, EPFL, Switzerland

2 Technische Universität Ilmenau, Germany

3 Structure \& Motion Laboratory, RVC, University of London, UK

${ }^{4}$ Chair of Biomechatronics, Technische Universität Ilmenau, Germany
}

are the phylogenetically older ones [8]. Although the spine movement adds a level of complexity to locomotion control, nature extensively uses spine movements at dynamic gaits, such as seen in the galloping cheetah. Theoretical research on the spine is rather well conducted, but we found only few hardware implementations. The spine can potentially contribute to energy storage, through its additional spine joints, and compliant elements. Such compliant elements also have the potential to produce thrust for the forward movement and extend the robot's stride length [9]. In this work we characterize the active, compliant spine in Lynx-robot (featuring the Cheetah-cub [4] leg design), in comparison to its predecessor Bobcat-robot [5]. All three robots were developed at the BIOROB laboratory, EPFL, Switzerland. We focus on the robot's interaction with the environment, and propose the following hypotheses:

1) The passive interaction between the environment and the robot due to in-series compliance of the spine contributes positively in achieving stable, open-loop locomotion-patterns with moderate speeds.

2) In this work we compare a directly actuated, singlejoint spine design (SV1) versus a spring-loaded, multijoint spine design (SV2 and SV3). Animals show a spine deflection, distributed as small deflections over many vertebrae. Hence we expect a higher performance of the suggested multi-joint spine design.

The current research centres around the bounding gait, a synchronous pattern with flight phases [14], [15]. Speed wise it is positioned between the half-bound and the gallop and may thus be considered a moderate speed gait. According to Hildebrand [16], [13] the stride circle describes a pairwise symmetric touchdown and lift-off of the front- and hind-feet, Figure 1. This enables easier implementation and testing of a

TABLE I: Comparison of selected quadruped robots with focus on bounding robots and robots with articulated spines: table data taken from [4] and [5], and extended by data from Lynx-robot; mass, robot height at hip-level, robot length, maximum speed, Froude number $\left(F R=\frac{\bar{v}^{2}}{g \cdot h}\right)$, body lengths/second, type of gait and presence of a spine.

\begin{tabular}{lllllllll} 
Robot & $\begin{array}{l}\mathrm{m}_{\text {rob }} \\
\mathrm{kg}\end{array}$ & $\begin{array}{l}\mathrm{h}_{\text {hip }} \\
\mathrm{m}\end{array}$ & $\begin{array}{l}\mathrm{l}_{\text {rob }} \\
\mathrm{m}\end{array}$ & $\begin{array}{l}\mathrm{v}_{\max } \\
\mathrm{m} \mathrm{s}^{-1}\end{array}$ & $\mathrm{FR}$ & $\begin{array}{l}\mathrm{BL} / s \\
\mathrm{~s}^{-1}\end{array}$ & Gait & Spine \\
\hline Scout II [3] & 20.865 & 0.323 & 0.552 & 1.3 & 0.53 & 2.4 & bound & no \\
BigDog [1] & 109 & 1 & 1.1 & 3.1 & 0.98 & 2.8 & bound & no \\
Puppy 1 [10] & 1.5 & 0.2 & 0.17 & 0.5 & 0.13 & 2.9 & bound & no \\
Puppy II [11] & 0.273 & 0.075 & 0.142 & 0.5 & 0.34 & 3.5 & bound & no \\
Takuma-quadruped [12] & 0.55 & 0.1 & 0.34 & 0.03 & 0.001 & 0.09 & walk & no \\
Cheetah-cub [4] & 1.1 & 0.158 & 0.205 & 1.42 & 1.30 & 6.9 & trot & no \\
Bobcat [5] & 1.03 & 0.125 & 0.166 & 0.78 & 0.5 & 4.7 & bound & yes \\
Lynx-SV1 & 1.2 & 0.154 & 0.224 & 0.75 & 0.25 & 3.3 & bound & yes \\
Lynx-SV2 & 1.2 & 0.154 & 0.226 & 0.6 & 0.24 & 2.7 & bound & yes \\
Lynx-SV3 & 1.2 & 0.154 & 0.225 & 0.6 & 0.24 & 2.7 & bound & yes
\end{tabular}




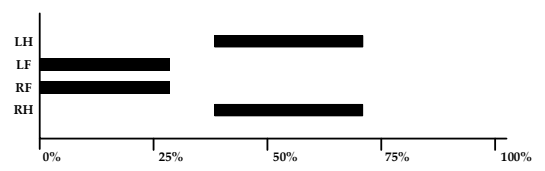

Fig. 1: Footfall patterns of a bound; LH=left-hind-foot, $\mathrm{LF}=$ left-fore-foot, RH and RF respectively (adapted from Hildebrand [13]).

prototype robot without additional destabilization due to the left-right-symmetry of the gait itself. Often shown is the use of the spine in the asymmetrical rotary gallop of the cheetah. It contributes to (1) widening the distance traveled during the flight-phase and also is partly responsible for the (2) overlapping of the feet during hind-stance and front-swing to achieve higher thrust. Hereby the spine of the cheetah bends differently in multiple points along its whole length. It can be observed that the vertical motion of the fore-trunk-segment rest minimal, so the animal has minimal disturbance of its different senses located in the head. The same mechanism of spine-movement is applicable for the bounding gait.

\section{EXPERIMENTAL PLATFORM LYNX-ROBOT, AND EXPERIMENTAL SETUP}

Lynx is a compliant quadruped robot with focus on multiple modular spine designs and a pantograph leg design (Figure 2). It is mainly built out of milled carbon- and glass fibre plates as well as 3D-printed ABS-pieces. The actuation is realized with RC-Servomotors (Kondo KRS2350 ICS, stall torque $2 \mathrm{Nm}$ at $6 \mathrm{~V}$ ) which are controlled by a RB110-electronics board with integrated Linux-OS. The robot has 9 actuated degrees of freedom (DOF), two per leg and one in the spine. It consists of two trunk segments of which the front one is slightly heavier (about $40 \mathrm{~g}$ ) caused by the location of the RB110, the legs and an active spine that connects the trunk elements. The spine-versions (SV) are all actively actuated but differ in their use of the compliant elements ( see sec. II-B, II-C and II-D) as well as a "single point of rotation" (strongest abstraction from nature) vs. "multiple point of rotation" (less strong abstraction from nature). The design is completed by a totally passive tail-like structure, which acts like a 5th-leg-stabilizer of the system in case of high pitching motion induced by bad gaits (it prevents the robot from falling backwards). In these cases, the compliant elements in the structure will push the robot in the opposite pitch-direction. This results in establishment of ground contact with all 4 legs. This taillike structure represents a non-bio-inspired part, as animals (expect the Kangaroo and some small mammals) seem not to use their tails for active pitch support during ground locomotion (ongoing research). The tail-like structure was added after initial trials and the reasoning will be explained later in this article. The specifications can be found in Table II.

\section{A. Leg-design}

The multi-segmented advanced spring loaded pantograph leg (ASLP) is mainly based on the Cheetah-cub leg design

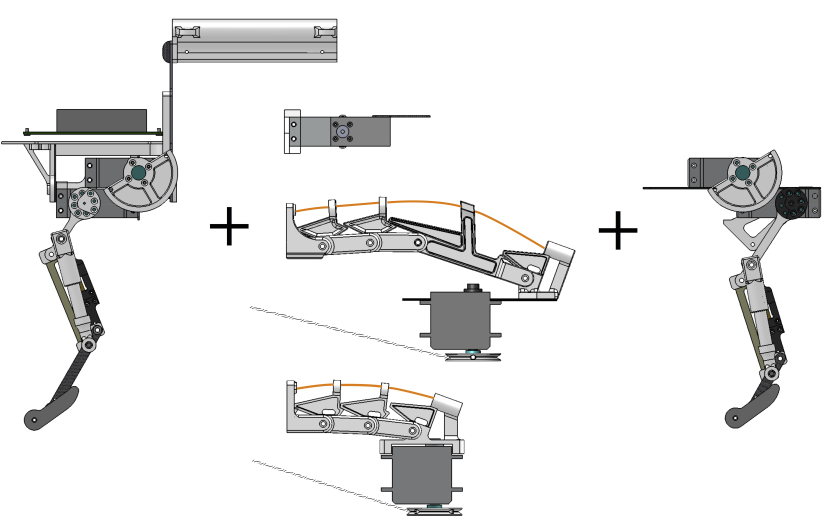

Fig. 2: Side view of Lynx with fore- and hind-trunk and all three exchangeable spine-modules, from top to bottom: SV1, SV2, SV3.

TABLE II: Hardware characteristics of Lynx-robot, spine version SV1, SV2, and SV3.

\begin{tabular}{l|ll} 
Parameter & Value & \\
\hline Mass & $1.2 \mathrm{~kg}$ & \\
Standing height & $0.154 \mathrm{~m}$ & \\
Width & $0.132 \mathrm{~m}$ & \\
Length & $0.224 \mathrm{~m}$ (SV1) 0.226 m (SV2) & $0.225 \mathrm{~m} \mathrm{(SV3)}$ \\
RC servo motor & Kondo KRS2350 ICS (9x) & \\
Control board & RoBoard RB110 & \\
Communication & Wifi card Via VT6655
\end{tabular}

and thus features a pantograph mechanism [17] with diagonal and second spring as well as a compliant foot element. The motors for hip and knee joints are located proximally (at the trunk/ analogous to biological paragons, cp. [18], [19]) to minimize the inertia of the leg and are connected with their respective actuation points either directly or through a cable mechanism. [4]

\section{B. Spine-design version 1 (SVI)}

Already used in Bobcat [5] this purely rotational spine is a simple but effective way of implementing a spinal undulation in the sagittal layer (upwards and downwards actively). It is actuated by one motor at its centre and has a in-series glass fibre rod as compliant element. This compliance prevents the motor to receive too high impacts during its oscillating motion and thus prolongs the lifetime of the motor. Important to know is, that the rotatory joint (here axis of the servo) is close to the front body segment. This stands in contrast to the animal world, where deflection over the whole length and not at a single rotational joint can be observed [9]. The exact point of rotation is subject of ongoing research, thus it is our interest to see if a very simplified spine can achieve the desired motion. [20] recently studied the influence of the rotation position and came to the conclusion that a position more to the rear could be beneficiary for dynamic robot locomotion.

\section{Spine-design version 2 (SV2)}

The second spine design (Figure 3 ) is purely composed of 3D-printed ABS pieces which are connected through steel axes. The structure seems more like that observed in nature 
because of the modular segments (equivalents of the "vertebrae"). It is able to move in the sagittal layer downwards actively (with RC-motor as flexor) and upwards until the blocking point passively (compliant rod as extensor). The specific shape allows a pre bending of the compliant element, again a glass fibre rod, which acts antagonistically to the actuation. The big difference of this design in comparison to $\mathrm{SV} 1$ lies in the passive reverse motion achieved through the spring element.
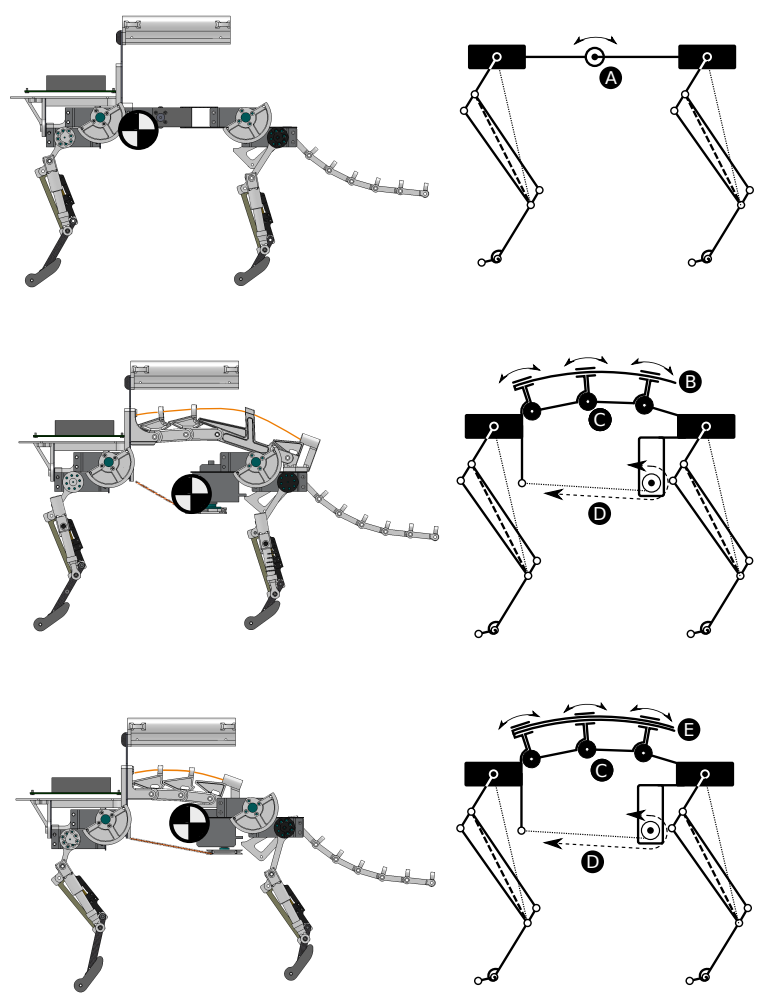

Fig. 3: Schematic presentation of Lynx-robot spine configurations, side-view, front to the left, computer-design (left) and schematic view (right). From top to bottom: SV1, SV2, and SV3. The markers on the computer design indicate the centre of mass for each configuration. (A) Single, rotatory, actuated joint of SV1. (B) Single leaf-spring, mounted in a pre-stressed fashion. (C) Multiple, passive, rotatory hinge joints of spine design SV2 and SV3; joints have limited range of rotation: only downwards, not upwards. (D) Antagonistic actuation based on pulley and cable mechanism, this actuation produces a flexing-torque of the SV2/SV3 spine. In case of external flexing forces, the cable mechanism goes slack. (E) Spine design SV3 applies two glass-fibre leaf springs in-parallel, and has a higher stiffness compared to SV2 (B). The symbol in the middle shows the position of the centre of mass (from CAD-model)

\section{Spine-design version 3 (SV3)}

$\mathrm{SV} 3$ is a multi-segmented spine build out of ABS (Figure 3), with structural similarities to SV2. Its passive elasticity consists of two glass fibre rods, in parallel. The rods are, due to the shape of the spine (mechanical stops at the equivalents of "vertebrae"), pre-bent and thus apply an upwards force . The actuation is achieved by one RC servo motor, which acts antagonistically to the glass fibre rods, by pulling via a string on a lever opposite to its mounting position. It is driven over a pulley to achieve straight alignment and to minimize effects that could influence stability. The biggest differences to SV2 are the stiffness of the spine, which is doubled, its length as well as the position of the contact with the hind trunk-segment.

\section{E. Locomotion Control}

The control of Lynx is realized through a parameterized, fully connected CPG-network ${ }^{1}$, running on the RB110 control board. Lynx-robot's CPG network consists of nine nonlinear oscillators (hip, knee for each leg and one for the spine) and, although possible, does not include any feedback (open-loop). With this control architecture a variety of gaits can be implemented by modifying three phase lag variables (hip-phase lags). Here a bound was chosen. That means, that the parallel legs act in-phase towards each other. The spinal actuation and thus its oscillator, is always phase-coupled to the left fore hip-joint (this is an arbitrary chosen joint for easy implementation of the control, coupling to a different joint and its impact should be researched in the future) and is treated as a virtual 5th hip joint with his own complete set of CPG control parameters (phase-lag, frequency, amplitude and offset). This also implies that we assume a coupling of the hip joints to the spine movement. To compute the necessary control signals for the motors (position controlled) we implemented forward kinematics, previously used in [4], [5]. The CPG network, which was previously used in Bobcat-robot [5], allows us to easily manipulate the main gait parameters, such as amplitudes and offsets of hips, spine and knees, duty factor (the time the foot remains in stance respective swing-phase) and the phase-relations of the actuators. By adjusting these key-parameters tests of the robots mechanics and a search for stable locomotion can be conducted.

\section{F. Experimental setup}

Kinematic data was recorded with 14 high-speed infrared cameras by Naturalpoint, Inc ${ }^{\circledR}$. The reflective markers on the robot were tracked in relation to the recording time with $f=250 \mathrm{~Hz}$. The positioning of cameras the in a rectangular shape, in a height of $1.5 \mathrm{~m}$ and $2.5 \mathrm{~m}$ around a catwalk allowed a coverage of a recording-volume with [width $=1 \mathrm{~m}$, length $=4 \mathrm{~m}$, height $=0.5 \mathrm{~m}$. The ground-plane was defined by calibration in advance of the experiments. The robot had to run in autonomous bound for $l=3 m \approx 13.3$ Bodylengths. The power-cable was held slack behind the robot. Naturalpoint Arena ${ }^{\circledR}$ was used to record, clean, trajectorize and save the motion data. Analysis (speed-derivation) was done with Mathworks ${ }^{\circledR}$ Matlab ${ }^{\circledR}$ and the b-tk plugin [22].

\footnotetext{
${ }^{1}$ Central pattern generators are “...neural circuits found in both invertebrate and vertebrate animals that can produce rhythmic patterns of neural activity without receiving rhythmic inputs..." [21]
} 


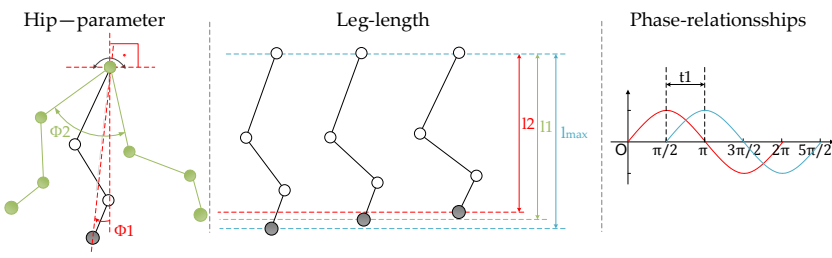

Fig. 4: Explanation of CPG-parameters for the legs: $\Phi_{1}$ is the hip-offset, $\Phi_{2}$ the hip-amplitude; $l_{1}$ the leg length offset; $l_{2}$ the leg length amplitude; $l_{\max }$ the maximum unbend leg length; $t_{1}$ presents the phase lag between two oscillators, such as the hip-oscillator and the leg-oscillators or the spine actuator. Offset of the spines is not shown: SV1 - middle position to reach a horizontal spine position; SV2 and SV3 - off-setted the way that the cable had no slack while in ground contact.

\section{RESUlts AND ANALYSis}

The tests with the three spine versions consisted of a grid search (the test-space for each spine-version was 180 different runs, Table III ). The robot was used for an extensive grid-search to find and optimize suitable and fast gaits. Simulation results would be faster to achieve, but suffer strongly from the given reality-gap due to simplifications of this mechanically complex system. The varied parameters can be found in the first part of Table III and produce a range of working and not working gaits. The spine offset for SV1 was set to reach a horizontal spine position (see Figure 3). The spines of SV2 and SV3 were off-setted, so the cable mechanism had no slack while on the ground and without movement. A list of the not varied CPG-parameters can be found in the second part of Table III.

TABLE III: First 6 rows: parameter space for the open, tested CPG-parameters. Last 7 rows: fixed CPG-parameterspace. 180 experiments per spine configuration were conducted. Please cp. Figure 4 for an explanation of the CPGparameters.

\begin{tabular}{l|l|l} 
CPG-parameter & Unit & Values \\
\hline Fore hip amplitude & $\mathrm{deg}$ & $40,50,60$ \\
Hind hip amplitude & $\mathrm{deg}$ & $30,40,50,60$ \\
Fore hip offset & $\mathrm{deg}$ & $20,25,30$ \\
Hind hip offset & $\mathrm{deg}$ & $15,20,25$ \\
Spine amplitude & $-1-0[]$ & $-0.2,-0.3,-0.4$ \\
Spine phase lag & $\mathrm{rad}$ & $0, \Pi / 2, \Pi$ \\
\hline Frequency & $\mathrm{Hz}$ & 2.5 \\
Virtual duty factor & {[]} & 0.3 \\
Leg-length-amplitude & $0-1[]$ & 0.6 \\
Leg-length stance deflection & $0-1[]$ & 0.0 \\
Leg-length offset & $0-1[]$ & 0.2 \\
Hip-leg phase lag & $\mathrm{rad}$ & 2.6 \\
Fore-hind phase lag & $\mathrm{rad}$ & $\Pi$
\end{tabular}

\section{A. Speed}

In contrast to gaits with no or even negative speeds $\left(v_{\min }=-0.58 \mathrm{~m} \mathrm{~s}^{-1}\right.$, Froude-Nr $\left.F R=0.23\right)$ due to wrong parameter combinations, the best gaits of SV1 produce up to $v_{\max }=0.75 \mathrm{~m} \mathrm{~s}^{-1} / F R=0.37$. Gaits that have a speed considered $v=0 \mathrm{~m} \mathrm{~s}^{-1}$ present a relatively big part in the results for the first spine design. It is especially visible for gaits having a spine phase lag of $\Pi$. The second design on the other hand shows in general gaits that have a positive speed $\left(v_{\min }=0.04 \mathrm{~m} \mathrm{~s}^{-1} / F R=0.001\right.$ to $v_{\max }=0.6 \mathrm{~m} \mathrm{~s}^{-1} /$ $F R=0.24)$. The last design, SV3 shows a speed range from $v_{\min }=0.05 \mathrm{~m} \mathrm{~s}^{-1} / F R=0.002$ to $v_{\max }=0.6 \mathrm{~m} \mathrm{~s}^{-1} /$ $F R=0.24$. SV2 and SV3 show a tendency in the speed to the positive and not close to 0 values, which indicates that the system are able to achieve locomotion in a moderate speed range with a broad set of control-parameters. SV1 in contrast exceeds very specific parameter combinations to locomote properly forwards.

TABLE IV: Varied CPG-parameters of the fastest gaits; FF/H-Amp/Off amplitudes and offsets of the hips, S-Amp/PL amplitude and phase-lag of the spine ; see Figure 4 for explanation of the CPG-parameters.

\begin{tabular}{l|llllll} 
& $\begin{array}{l}\text { F-H-Amp } \\
{[\mathrm{deg}]}\end{array}$ & $\begin{array}{l}\text { H-H-Amp } \\
{[\mathrm{deg}]}\end{array}$ & $\begin{array}{l}\text { F-H-Off } \\
{[\mathrm{deg}]}\end{array}$ & $\begin{array}{l}\text { H-H-Off } \\
{[\mathrm{deg}]}\end{array}$ & $\begin{array}{l}\text { S-Amp } \\
{[-1-0[]]}\end{array}$ & $\begin{array}{l}\text { S-Pl } \\
{[0-\Pi]}\end{array}$ \\
\hline SV1 & 50 & 50 & 30 & 25 & -0.4 & 0 \\
SV2 & 60 & 60 & 20 & 15 & -0.3 & $\Pi$ \\
SV3 & 60 & 60 & 20 & 15 & -0.2 & $\Pi$
\end{tabular}

\section{B. Gait-classification}

The classification of "natural looking" animal gaits in robots can be done by considering two major points. First the footfall pattern, which is characteristic for each gait and second the vertical position change of the trunk. Figure 5 shows the stride-cycle of the Lynx-Versions highest speed gaits as well as the respective (qualitatively from video derived) footfall-patterns in combination with the derived duty factors. SV2 has the strongest resemblance with the footfall-pattern seen in Figure 1, the animal-like bound. It is followed by SV1, which lacks flight phases and in addition makes use of the tail-like structure to be able to move at all (this makes the design less desirable for bio-inspired cat- or dog-like robots). SV3 shows overlapping foot contact with fore and hind feet and thus a duty-factor over 0.5. This is not the case in a animal-like bound. The results of the motionanalysis (Figure 6) partly confirm these findings, by stating the lowest average vertical position change for SV2 followed by SV3 and SV1 (SV1: $d_{a v}=0.11 \mathrm{~m}$, SV2: $d_{a v}=0.06 \mathrm{~m}$ and SV3: $\left.d_{a v}=0.07 \mathrm{~m}\right)$. Its large vertical position changes explain as well, why SV1 was the only version, that made active use of the stabilizing structure to be even able to move.

In Fig. 7 it is visualized, that the second spine design (SV2) has the highest number of natural looking gaits. This is due to the fact, that SV1 has more gaits with very high pitch angles, that were able to produce fast movement due to correction effects of the tail-like structure. These gaits on the other hand do not resemble a bound as observed in nature, but a kind of artificial gait. SV3 shows gaits that look quite natural, but due to the high stiffness show even less flight phases than the other two. SV2 is able to adapt to the environment and misalignment during the movement nicely and thus looks natural in general. Here is shown that a 

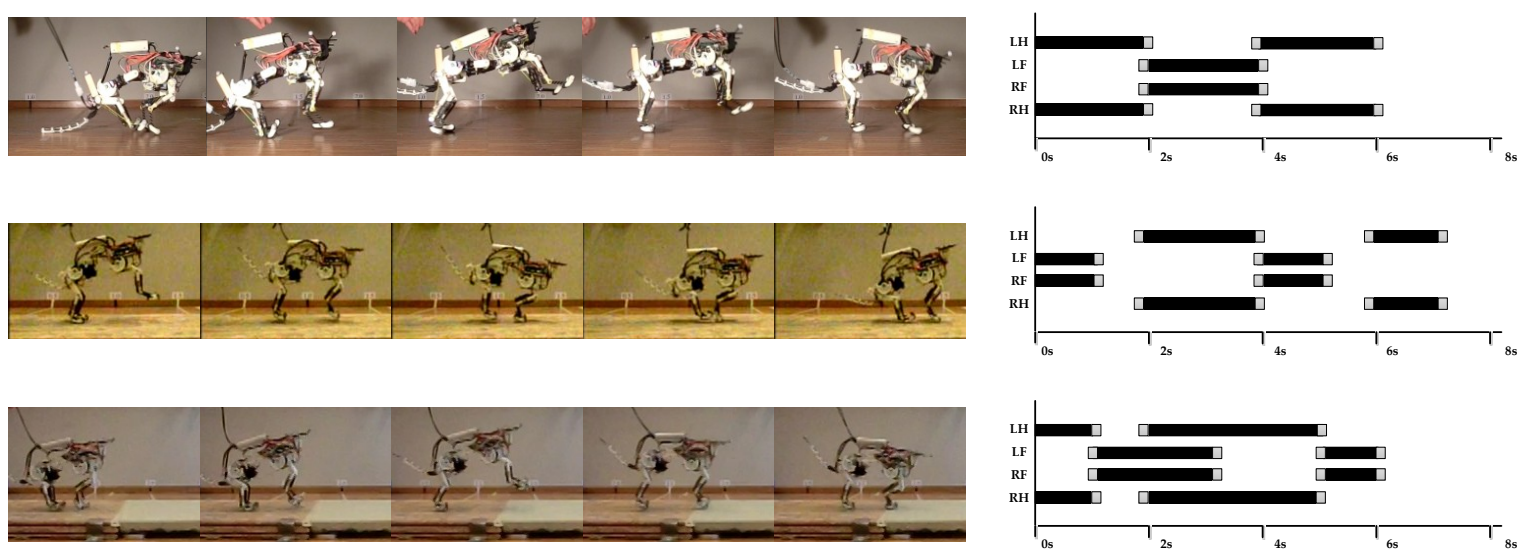

Fig. 5: Representative bound-gait snapshots (left) and corresponding qualitative from video derived footfall-patterns (right/grey: error-margin due to optical videoanalysis) of the fastest gaits of the three tested Lynx-robot spine configurations (SV1, SV2, SV3, from top to bottom, respectively). SV1: $v=0.75 \mathrm{~m} / \mathrm{s}, \mathrm{SV} 1$ is the only configuration that required stabilization in pitch-rotation, via its tail-like structure preventing falling backwards (visible in the first snapshot/ strong influence on gait), real Duty-factor (relation of stance to swing phase of the legs) $D F_{a v}=0.5$. SV2: $v=0.6 \mathrm{~m} / \mathrm{s}$, and no ground contact of its tail-like structure, real Duty-factor $D F_{a v}=0.4$. SV3: $v=0.6 \mathrm{~m} / \mathrm{s}$, no ground contact of tail-like structure, real Duty-factor $D F_{a v}=0.625$.

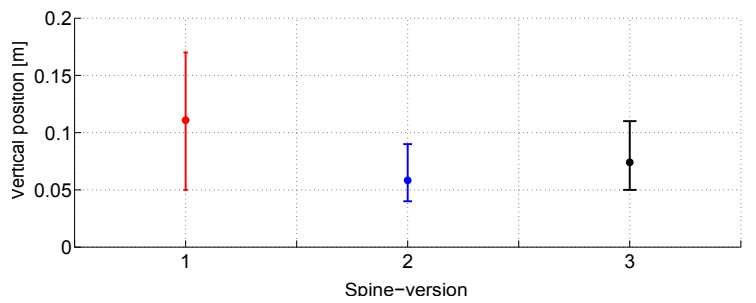

Fig. 6: Change of the instantaneous, vertical position of the robot trunk, for the best 10 gaits per spine configuration.: $\left(\mathrm{SV} 1: d_{a v}=0.11 \mathrm{~m}\right),\left(\mathrm{SV} 2: d_{a v}=0.06 \mathrm{~m}\right)$ and $\left(\mathrm{SV} 3: d_{a v}=\right.$ $0.07 \mathrm{~m}$ ). Lynx-robot in SV1 applied gaits with much higher vertical excursion; between $5 \mathrm{~cm}$ and up to $17 \mathrm{~cm}$, compared to SV2. The high vertical jumps of SV1 indirectly led to a higher maximum robot speed, but would have completely destabilized the robot without its strut-like structure in the back of the robot (see also Figure 5).

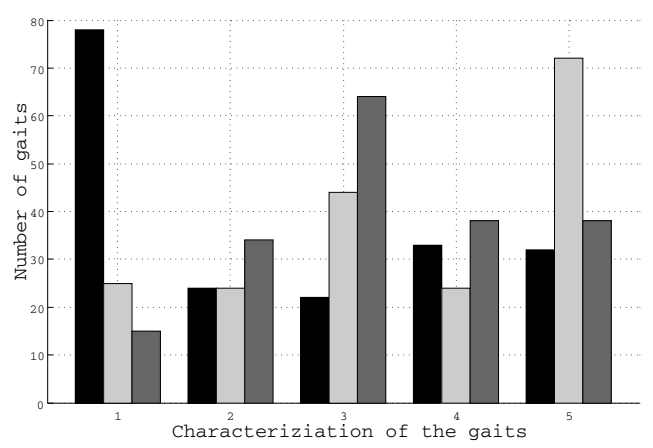

Fig. 7: Distribution of "non-natural" and "natural" looking gaits: black—SV1, light grey-SV2, dark grey-SV3; scale (x-axis) from 1 (non-natural) to 5 (natural) wide parameter range can be used to produce feasible gaits. This emphasizes the adaptability to the environment due to elasticity in the spine.

\section{Discussion AND Future WORK}

TABLE V: Speed comparison of the best gaits in all version with respective Bobcat-gait; first: actual speed, second: Froude number (data taken from [5]).

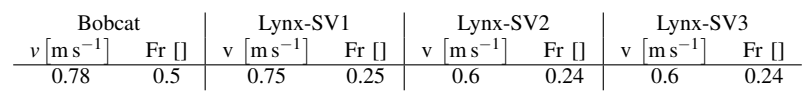

The spine of SV1 is identical to the one used for Bobcat and enables the comparison of two robots with only small difference in size and different leg-designs(3-segmented in Lynx and 2-segmented in Bobcat). The robots show almost the same top speed. The spine versions 2 and 3 are $\approx 21 \%$ slower than SV1, $\approx 25 \%$ and slower than Bobcat. That results in a Froude-Nr for Lynx that is overall half the one of Bobcat due to differences in leg-length. This points to an advantage if using a two-segmented leg in combination with a simple, rotational spine as well as the need for complexer spines as soon as the leg design represents biology more closely. The difference in speed is due to the use of a different spine architecture with higher elasticity. The shift from a single, to a multi-rotation point of the spine provided more stable locomotion. As shown in the Section III-B the multisegmented spines, with the right level of stiffness, seem to enable the robot to move more with bound-characteristics found in literature, such as flight-phases in the footfallpattern as well as pitch stability and acceptance of a wider range of control parameters (confirming hypothesis 1). The single-rotation spine in SV1 might thus be too strongly 
abstracted in comparison with the multi-vertebrae spine in the long-spined animal role models, if used in combination with the ASLP-leg (Bobcat-robot manages quite nicely). Although SV3 shows comparable results in the top speed, it differs in the observed characteristics of the gaits in SV2. The reason for this might be the slower reaction time of the spine, due to higher spine-stiffness, and the resulting delay in the flexion of the spine. Hyposthesis 2 stated an advantage in overall performance, which could not be shown in this work. The likely reasons for this are the improvements that have to be done in the robot-design itself: Center of mass should be shifted lower and more to the front; the direct production of the spine movement in SV1 seems (in our case) to be more effective and should be combined with the stabilizing effects achieved through the multi-segmented spines in SV2 and SV3. Based on the observations, new insight into the mechanical design of a compliant spine in combination with advanced spring loaded pantograph legs was gained and thus should be implemented in future versions. Also, there is the need to explore an even wider parameter space by building a detailed model of the robot in simulation and refining the gaits more. The impact of how to integrate the spinal controller in the general framework should be investigated further as coupling to a different oscillator in the CPGnetwork could play an important role. The observed use of the tail-like structure and its need as a stabilizing element in SV1 is as well an interesting topic for current investigation. As we used spines with different levels of abstraction (single rotation/ u-like structure), a decrease of this abstraction with an s-like spine and supportive active flexion/extension could be the next step to develop an agile system. Additional animal data is being analyzed to find the optimal grade of biomimicri vs. bio-inspiration.

\section{CONCLUSiON}

We observed, that the use of a compliant spine in combination with the ASLP-leg-design can produce reasonably fast and stable bounding gaits. Different spine-designs result hereby in very different motion patterns. In contrast to the natural multi-vertebrae structure, a strong abstraction in a single-rotational-spine resulted in more artificial gait patterns with strong instability problems, but fast movement.

\section{ACKNOWLEDGMENT}

We thank Alexandre Tuleu for providing the code of the CPG-network, Mahdi Khoramshahi for MatLab ${ }^{\circledR}$ advice and Massimo Vespigninai for providing very useful comments on the article. The research leading to these results has received funding from the European Communitys Seventh Framework Programme (FP7/2007-2013-Challenge 2-Cognitive Systems, Interaction, Robotics; grant agreement number 248311 (AMARSi)) and from the Swiss National Science Foundation through the National Centre of Competence in Research Robotics.

\section{REFERENCES}

[1] M. Raibert, K. Blankespoor, G. Nelson, R. Playter, et al., "Bigdog, the rough-terrain quadruped robot," in Proceedings of the 17th World Congress The International Federation of Automatic Control, 2008, pp. 10823-10825.

[2] K. Tsujita and K. Miki, "A study on trunk stiffness and gait stability in quadrupedal locomotion using musculoskeletal robot," in Advanced Robotics (ICRA), 2011 15th International Conference on, 2011, pp. 316-321.

[3] I. Poulakakis, J. A. Smith, and M. Buehler, "Modeling and Experiments of Untethered Quadrupedal Running with a Bounding Gait: The Scout II Robot," The International Journal of Robotics Research, vol. 24, no. 4, pp. 239-256, Apr. 2005.

[4] A. Spröwitz, A. Tuleu, M. Vespignani, M. Ajallooeian, E. Badri, and A. Ijspeert, "Towards Dynamic Trot Gait Locomotion-Design, Control, and Experiments with Cheetah-cub, a Compliant Quadruped Robot," The International Journal of Robotics Research, vol. 32, no. 8, pp. 932-950, 2013.

[5] M. Khoramshahi, A. Spröwitz, A. Tuleu, M. Ahmadabadi, and A. Ijspeert, "Benefits of an Active Spine Supported Bounding Locomotion With a Small Compliant Quadruped Robot," in Proceedings of 2013 IEEE International Conference on Robotics and Automation, 2013.

[6] H.-W. Park, M. Y. M. Chuah, S. Kim, et al., "Quadruped bounding control with variable duty cycle via vertical impulse scaling," in The 2014 IEEE/RSJ International Conference on Intelligent Robots and Systems, Chicago, Illinois, 2014.

[7] H. Witte, M. Fischer, N. Schilling, W. Ilg, R. Dillmann, M. Eckert, and J. Wittenburg, "Konstruktion vierbeiniger laufmaschinen," Konstruktion, vol. 52, no. 9, pp. 46-50, 2000.

[8] M. S. Fischer and H. Witte, "Legs evolved only at the end!" Philosophical Transactions of the Royal Society A: Mathematical, Physical and Engineering Sciences, vol. 365, no. 1850, pp. 185-198, 2007.

[9] J. E. Bertram and A. Gutmann, "Motions of the running horse and cheetah revisited: fundamental mechanics of the transverse and rotary gallop," Journal of The Royal Society Interface, vol. 6, no. 35, pp. 549-559, 2009.

[10] F. Iida and R. Pfeifer, "Cheap rapid locomotion of a quadruped robot: Self-stabilization of bounding gait," in Intelligent Autonomous Systems, vol. 8, 2004, pp. 642-649.

[11] F. Iida, G. Gomez, and R. Pfeifer, "Exploiting body dynamics for controlling a running quadruped robot," in 12th International Conference on Advanced Robotics, 2005. ICAR '05. Proceedings. IEEE, July 2005, pp. 229-235.

[12] T. Takuma, M. Ikeda, and T. Masuda, "Facilitating multi-modal locomotion in a quadruped robot utilizing passive oscillation of the spine structure," in Intelligent Robots and Systems (IROS), 2010 IEEE/RSJ International Conference on, 2010, pp. 4940-4945.

[13] M. Hildebrand, "The quadrupedal gaits of vertebrates," BioScience, vol. 39, no. 11, pp. pp. 766-775, 1989.

[14] J. J. Robilliard, T. Pfau, and A. M. Wilson, "Gait characterisation and classification in horses," Journal of Experimental Biology, vol. 210, no. 2, p. 187, 2006.

[15] M. B. Bennett, Tetrapod Walking and Running. John Wiley and Sons, Ltd, 2001.

[16] M. Hildebrand, "Analysis of asymmetrical gaits," Journal of Mammalogy, vol. 58, no. 2, pp. pp. 131-156, 1977.

[17] H. Witte, R. Hackert, M. Fischer, W. Ilg, J. Albiez, R. Dillmann, and A. Seyfarth, "Design criteria for the leg of a walking machine derived by biological inspiration from quadrupedal mammals," in Proceedings CLAWAR2001-4th International Symposium on Climbing and Walking Robots, 2001, pp. 63-68.

[18] M. Hildebrand, "Walking and running," Functional vertebrate morphology, vol. 3, pp. 38-57, 1985.

[19] M. Hildebrand and J. P. Hurley, "Energy of the oscillating legs of a fast-moving cheetah, pronghorn, jackrabbit, and elephant," Journal of morphology, vol. 184, no. 1, pp. 23-31, 1985.

[20] Q. Zhao, H. Sumioka, and R. Pfeifer, "The effect of morphology on the spinal engine driven locomotion in a quadruped robot," in The 5th International Symposium on Adaptive Motion of Animals and Machines (AMAM2011), 2011, pp. 51-52.

[21] A. J. Ijspeert, "Central pattern generators for locomotion control in animals and robots: a review," Neural Networks, vol. 21, no. 4, pp. 642-653, 2008.

[22] A. Barre and S. Armand, "b-tk biomechanical toolkit," 2011a. 